19.

\title{
Ueber die Transformationen, welche in der Varia- tionsrechnung zur Nachweisung grölster oder kleinster Werthe dienen.
}

(Von Herrn Minding zu Dorpat.)

Die von Jacobi im $17^{\text {ten }}$ Bande dieses Journals S. 71 in die Variationsrechnung eingeführten Transformationen, die seitdem schon mehrmals, namentlich noch jüngst in dieser Zeitschrift von Herrn E. Heine, behandelt worden sind, lassen sich in vollständig entwickelter Gestalt aus einem algebraischen Lehrsatze herleiten, der in folgender Formel enthalten ist, nämlich:

$$
\text { (A.) } \sum_{j}(b-j)_{j}\left\{(a-b+j)_{v-j}+(a-b+j-1)_{v-j-1}\right\}=a_{v} \text {. }
$$

In dieser Formel sind $a$ und $b$ beliebige, $v$ und $j$ ganze Zahlen; $a_{v}$ bedeutet in üblicher Weise die Vorzahl von $x^{\nu}$ in der Entwicklung von $(1+x)^{a}$ und mufs daher immer gleich 0 gesetzt werden, wenn $\nu$ negativ ist; die Summation nach $j$ umfafst alle Werthe, welche gültige (d. h. nicht verschwindende) Glieder geben; der Anblick der Formel lehrt, dafs diese Werthe von $j$ weder selbst negaliv sein, noch $\nu-j$ negativ machen dürfen. Die Formel (A.) ist daher richlig, wenn $\nu$ negativ ist; sie ist auch richtig für $\nu=0$, denn alsdann erhält man rechter Hand 1 und linker Hand nur ein gültiges Glied für $j=0$, dessen Werth ebenfalls 1 ist. Der Beweis ist also nur noch für ein positives ganzes $\nu$ zu führen.

Zur Abkürzung setze man die im Folgenden häufig auftretende Form $(-a)_{i}+(-a-1)_{i-1}=f(a, i)$, also:

$$
f(a, i)=(-a)_{i}+(-a-1)_{i-1}=(-1)^{i}\left\{(a+i-1)_{i}-(a+i-1)_{i-1}\right\} ;
$$

so ist für ein negatives $i$ (welches übrigens hier immer nur eine ganze Zahl sein kann), $f(a, i)=0$; für $i=0$ wird $f(a, 0)=1$; für ein positives $i$ ist:

$$
f(a, i)=(-1)^{i} \frac{(a-i)(a+1)(a+2) \ldots(a+i-1)}{1.2 .3 \ldots i} .
$$

Auch gilt folgender Satz: Sind $m$ und $i$ ganze Zahlen, deren Summe positiv ist, so ist:

$$
(-1)^{m} f(m, i)+(-1)^{i} f(i, m)=0
$$


hingegen für $m+i=0$ wird vorstehende Summe $=(-1)^{m} 2$. Es folgt hieraus $f(m, m)=0$, wenn $m$ positiv ist; für ein negatives $m$ ist ebenfalls $f(m, m)=0$; für $m=0$ aber wird $f(0,0)=1$.

Um nun die Formel (A.) zu beweisen, werde

$$
\sum_{j}(b-j)_{j} f(b-a-j, v-j)-a_{v}=\varphi(a, b, v)
$$

gesetzt; so ist zu zeigen, dafs $\varphi(\boldsymbol{a}, \boldsymbol{b}, \nu)$ auch für jedes positive ganze $\nu$, wie für negative $\nu$ und für $\nu=0$ verschwindet, welche Werthe auch $a$ und $b$ haben mögen. Schreibt man für $v, v-i$ und für $b, b-i$, wo $i$ eine ganze Zahl bedeutet, so erhält man:

$$
\sum_{j}(b-i-j)_{j} f(b-a-i-j, \nu-i-j)=a_{\nu-i}+\varphi_{i},
$$

wo zur Abkürzung $\varphi_{i}$ für $\varphi(a, b-i, \nu-i)$ gesetzt ist. Diese Gleichung werde mit $f(b, i)$ multiplicirt und die Summe der Producte für alle $i$ genommen, welche gültige Glieder geben, oder auch, ohne solche $i$ vorläufig zu unterscheiden, für alle $i$ von $-\infty$ bis $+\infty$. Es entsteht so die folgende Gleichung:

$$
\sum_{i} \sum_{j} f(b, i)(b-i-j)_{j} f(b-a-i-j, v-i-j)=\sum_{i} f(b, i)\left\{a_{\nu-i}+\varphi_{i}\right\} .
$$

Wird hier $j=\mu-i$ gesetzt, so verwandelt sich diese Formel in nachstehende:

$$
\sum_{i} \sum_{\mu} f(b, i)(b-\mu)_{\mu-i} f(b-a-\mu, \nu-\mu)=\sum_{i} f(b, i)\left\{a_{\nu-i}+\varphi_{i}\right\},
$$

worin die Summation nach $i$ sogleich ausführbar ist; man hat nämlich nach einem bekannten Satze:

$$
\begin{aligned}
\sum_{i} f(b, i)(b-\mu)_{\mu-i} & =\sum_{i}(-b)_{i}(b-\mu)_{\mu-i}+\sum_{i}(-b-1)_{i-1}(b-\mu)_{\mu-i} \\
& =(-\mu)_{\mu}+(-\mu-1)_{\mu-1}=f(\mu, \mu) .
\end{aligned}
$$

Es ist aber $f(\mu, \mu)=1$, wenn $\mu=0$, in allen anderen Fällen ist $f(\mu, \mu)=0$; folglich ist auf der linken Seite die Summation nach $\mu$ nicht weiter vorzunehmen, sondern nur $\mu=0$ einzusetzen, wodurch die zweifache Summe linker Hand den Werth $f(b-a, v)$ erhält. Denselben Werth giebt aber auch die Summe:

$$
\begin{aligned}
\sum_{i} f(b, i) a_{\nu-i} & =\sum_{i}(-b)_{i} a_{\nu-i}+\Sigma(-b-1)_{i-1} a_{\nu-i} \\
& =(a-b)_{\nu}+(a-b-1)_{\nu-1}=f(b-a, v) ;
\end{aligned}
$$

daher ist $\sum_{i} f(b, i) \varphi_{i}=0$.

Nun ist aber bereits bekannt, dafs $\varphi_{i}=0$, wenn $\nu-i$ negativ oder auch gleich Null ist; ferner ist $f(b, i)=0$, wenn $i$ negativ; daher darf vor- 
stehende Gleichung, mit Weglassung der sich unmittelbar als ungültig ankündigenden Glieder, also geschrieben werden:

$$
\sum_{i=0}^{i=v-1} f(b, i) \varphi(a, b-i, v-i)=0 .
$$

Wird hier nach und nach $v=1,2,3, \ldots$ eingesetzt und beachtet, dafs $f(b, 0)=1$, so folgt:

für $\nu=1 \quad \varphi(a, b, 1)=0$,

für $v=2 \quad \varphi(a, b, 2)+f(b, 1) \cdot \varphi(a, b-1,1)=0$,

für $v=3 \varphi(a, b, 3)+f(b, 1) \cdot \varphi(a, b-1,2)+f(b, 2) \cdot \varphi(a, b-2,1)=0$,

u. s. w.

Die erste ${ }^{\prime}$ Gleîchung $\varphi(a, b, 1)=0$ gilt aber für jedes $b$, also ist auch $\varphi(a, b-1,1)=0$ und folglich $\varphi(a, b, 2)=0$ wiederum für jedes $b$; also $\varphi(a, b-2,1)=0$ und $\varphi(a, b-1,2)=0$, daher $\varphi(a, b, 3)=0$, u. s. f. allgemein $\varphi(\boldsymbol{a}, \boldsymbol{b}, v)=0$ für jedes positive ganze $\nu$; wodurch die Gleichung (A.) erwiesen ist. Schreibt man darin $\beta+\nu$ für $b$, so kann sie auch leicht auf folgende Gestalt gebracht werden:

$\sum_{j=0}^{i=\nu-1}(\beta+\nu-j)_{j} \frac{(a-\beta)(a-\beta-\nu+j-1)(a-\beta-\nu+j-2) \ldots(a-\beta-2 \nu+2 j+1)}{1.2 .3 . \nu-j}=a_{\nu}-\beta_{\nu}$, wo alle Glieder der linken Seite durch $a-\beta$ theilbar sind, so dafs die Formel den Quotienten $\frac{\alpha_{\nu}-\beta_{\nu}}{\alpha-\beta}$ auf eine besondere Weise darstellt; ich bleibe jedoch bei ihrer ersten Gestalt stehen, um noch einige Zusätze daran zu knüpfen.

Wird die Formel (A.) mit $x^{\nu}$ multiplicirt und die Summe der Producte von $v=0$ bis $\nu=\infty$ genommen, so folgt:

$$
\sum_{\nu} \sum_{j}(b-j)_{j} f(b-a-j, v-j) x^{\nu}=\Sigma a_{\nu} x^{\nu}=(1+x)^{a} .
$$

Vertauscht man hier $\nu$ mit $i+j$, so geht diese Gleichung über in:

Es ist aber:

$$
\sum_{i} \sum_{j}(b-j)_{j} f(b-a-j, i) x^{i+j}=(1+x)^{a} \text {. }
$$

$\sum_{i} f(b-a-j, i) x^{i}=(1+x)^{a-b+j}+x(1+x)^{a-b+j-1}=(1+x)^{a-b+j-1}(1+2 x)$; dies giebt nach Aufhebung des gemeinsamen Factors $(1+x)^{a}$ die Formel:

$$
\sum_{j}(b-j)_{j}\{x(1+x)\}^{j}=\frac{(1+x)^{b+1}}{1+2 x}
$$

oder wenn $4 x(1+x)=z, 2(1+x)=1+\sqrt{1+z}$ gesetzt und $a$ für $b+1$ geschrieben wird, so erhält man folgende Reihe: 


$$
\begin{gathered}
\frac{(1+\sqrt{1+z})^{a}}{\sqrt{1+z}}= \\
2^{a}\left\{1+(a-2) \frac{z}{4}+\frac{(a-3)(a-4)}{1.2}\left(\frac{z}{4}\right)^{2}+\frac{(a-4)(a-5)(a-6)}{1.2 .3}\left(\frac{z}{4}\right)^{3}+\cdots\right\}
\end{gathered}
$$

Setzt man in der Reihe (A.) für $b$ den Werth $\nu$, multiplicirt wieder mit $x^{v}$ und addirt nach $\nu$, so entsteht:

$$
\left.\sum_{\nu} \sum_{j}(\nu-j)_{j} f(\nu-a-j), \nu-j\right) x^{\nu}=\Sigma a_{\nu} x^{\nu}=(1+x)^{a} .
$$

Wird hier wieder $i+j$ für $v$ eingeführt, so erhält man auf der linken Seite:

$$
\sum_{i} \sum_{j} i_{j} f(i-a, i) x^{i+j}
$$

oder weil $\sum_{j} i_{j} x^{j}=(1+x)^{i}$,

$$
\sum_{i} f(i-a, i)\{x(1+x)\}^{i}=(1+x)^{a},
$$

d. h. Wenn $z$ die vorige Bedeutung behält:

$$
(1+\sqrt{1+z})^{a}=2^{a}\left\{1+a \frac{z}{4}+\frac{a(a-3)}{1.2}\left(\frac{z}{4}\right)^{2}+\frac{a(a-4)(a-5)}{1.2 .3}\left(\frac{z}{4}\right)^{3}+\cdots\right\} .
$$

Es sei jetzt a eine positive ganze Zahl $n$, und wieder $b$ gleich $v$, so giebt die Formel (A.):

$$
\sum_{j}(\nu-j)_{j} f(\nu-n-j, v-j)=n_{\nu}
$$

Multiplicirt man diese Gleichung mit $x^{\nu}$ und summirt von $\nu=0$ bis $\nu=n$, so erhält man auf der rechten Seite $(1+x)^{n}$; setzt man auf der linken wieder $\nu=j+i$, so entsteht die Formel:

$$
\sum_{i} \sum_{j} i_{j} f(i-n, i) x^{i+j}=(1+x)^{n},
$$

worin jedoch nur solche positive Werthe von $i$ und $j$ gültig sind, welche $i+j$ höchstens gleich $n$ geben. Es wird aber für $i=n, f(i-n, i)=f(0, n)=(-1)^{n-1}$, und zu $i=n$ gehört der Werth 0 von $j$; also entsteht für $i=n, j=0$ in vorstehender Summe das Glied $(-1)^{n-1} x^{n}$. Ferner ist:

$$
f(i-n, i)=(n-i)_{i}+(n-i-1)_{i-1}=0,
$$

wenn $n-2 i$ negativ ist; folglich beschränken sich die gültigen Werthe von $i$ auf die Reihe $1,2,3, \ldots n^{\prime}$, wenn $n^{\prime}$ die gröfste in $\frac{n}{2}$ enthaltene ganze Zahl bedeutet, und da $i_{j}=0$ wird, wenn $j>i$ ist, so folgt:

$$
\sum_{j=0}^{j=n} i_{j} x^{j}=\sum_{j=0}^{j=i} i_{j} x^{j}=(1+x)^{i}
$$


daher erhält man:

oder

$$
\sum_{i=0}^{i=n^{\prime}} f(i-n, i)\{x(1+x)\}^{i}+(-1)^{n-1} x^{n}=(1+x)^{n}
$$

$$
\begin{aligned}
(1+x)^{n}+(-1)^{n} x^{n}=1+ & n x(1+x)+\frac{n(n-3)}{1.2}\{x(1+x)\}^{2}+\cdots \\
& +\frac{n\left(n-n^{\prime}-1\right)\left(n-n^{\prime}-2\right) \ldots\left(n-2 n^{\prime}+1\right)}{1.2 .3 \ldots n^{\prime}}\{x(1+x)\}^{n^{\prime}} .
\end{aligned}
$$

Alle diese übrigens längst bekannten Formeln ergeben sich, wie man sieht, mit grofser Leichtigkeit aus der Grundgleichung (A.), was zu zeigen der Mühe werth schien; für den gegenwärtigen Zweck bedarf es ihrer jedoch nicht, wohl aber einer anderen Formel, die der zuletzt entwickelten ganz analog ist und ebenso wie diese aus der Grundgleichung abgeleitet werden kann.

Sind nämlich $v$ und $w$ zwei Functionen von $x$, so ist $\frac{d^{n}(v w)}{d x^{n}}$ oder, wie ich kürzer schreibe:

$$
d^{n}(v w)=\sum_{\nu} n_{\nu} v^{(n-\nu)} w^{(\nu)}
$$

also

$$
\left.d^{n}(v w)=\sum_{\nu} \sum_{j}(\nu-j)_{j} f^{\prime} \nu-n-j, v-j\right) \cdot v^{(n-v)} w^{(v)}
$$

oder, $\nu-j=i$ gesetzt:

$$
d^{n}(v w)=\sum_{i} \sum_{j} i_{j} f(i-n, i) \cdot v^{(n-j-i)} w^{(j+i)}
$$

wo die Summation alle positiven Werthe von $i$ und $j$ umfafst, für welche $\boldsymbol{i}+\boldsymbol{j} \overline{\bar{n}}$ ist. Hieraus folgt aber ebenso wie vorhin:

$$
d^{n}(v w)=\sum_{i=0}^{i=n^{\prime}} \sum_{j} i_{j} f(i-n, i) \cdot v^{(n-j-i)} w^{(j+i)}+(-1)^{n-1} v \cdot w^{(n)},
$$

unter der Bedingung $i+j \bar{\sum}$. Es ist aber:

$$
\sum_{j=0}^{j=n-i} i_{j} w^{(j+i)} v^{(n-j-i)}=d^{i}\left(v^{(n-2 i)} w^{(i)}\right)
$$

daher folgt:

oder:

$$
\text { (B.) } \quad d^{n}(v w)=\sum_{i=0}^{i=n^{\prime}} f(i-n, i) d^{i}\left(v^{(n-2 i)} w^{(i)}\right)+(-1)^{n-1} v \cdot w^{(n)}
$$

$$
\begin{aligned}
d^{n}(v w)+(-1)^{n} v \cdot w^{(n)}= & v^{(n)} u+n d\left(v^{(n-2)} w^{\prime}\right)+\frac{n(n-3)}{1.2} d^{2}\left(v^{(n-4)} w^{\prime \prime}\right)+\cdots \\
& +\frac{n\left(n-n^{\prime}-1\right)\left(n-n^{\prime}-2\right) \ldots\left(n-2 n^{\prime}+1\right)}{1.2 .3 \ldots n^{\prime}} d^{n^{\prime}}\left(v^{\left(n-2 n^{\prime}\right)} w^{n^{\prime}}\right) .
\end{aligned}
$$


Mit Hülfe der Formeln (A.) und (B.) lassen sich nun die Jacobischen Sätze auf folgende Art herleiten:

Der erste Satz kommt fast unmittelbar darauf zurück, dafs ein Ausdruck $A$ von der Form:

$$
\Delta=y d^{m}\left(\mathfrak{A}(y \cdot t)^{(m)}\right)-y t d^{m}\left(\mathfrak{A} y^{(m)}\right)
$$

sich auf nachstehende Gestalt bringen läfst:

$$
\Delta=d\left(\mathfrak{B}_{1} t^{\prime}\right)+d^{2}\left(\mathfrak{B}_{2} t^{\prime \prime}\right)+\cdots+d^{m}\left(\mathfrak{B}_{m} t^{(m)}\right),
$$

wo die $\mathfrak{B}$ durch $\mathfrak{A}, \boldsymbol{y}$ und deren Ableitungen nach $\boldsymbol{x}$ ausgedrückt werden, von $t$ aber unabhängig sind.

Es ist $(y t)^{(m)}$ oder $d^{m}(y t)=\sum_{\mu} m_{\mu} y^{(m-\mu)} t^{(\mu)}$, folglich:

$y \cdot d^{m}\left(\mathfrak{A}(y \boldsymbol{t})^{(m)}\right)=\sum_{\mu} m_{\mu} y d^{m}\left(\mathfrak{A} y^{(m-\mu)} \boldsymbol{t}^{(\mu)}\right)=\sum_{\mu} \sum_{\varepsilon} m_{\mu} m_{\varepsilon} y \boldsymbol{t}^{(\mu+\varepsilon)} d^{m-\varepsilon}\left(\mathfrak{A} y^{(m-\mu)}\right)$. Werden hier alle Glieder zusammengefafst, für welche $\mu+\varepsilon$ denselben Werth $\lambda$ háa, so erhält man:

$$
y d^{m}\left(\mathfrak{A}(y t)^{(m)}\right)=\sum_{\lambda} \mathfrak{R}_{\lambda} t^{\lambda}=\Delta+\mathfrak{\Omega}_{0} t,
$$

wo $\mathfrak{l}_{\lambda}=\sum_{\varepsilon} \boldsymbol{m}_{\varepsilon} \boldsymbol{m}_{\lambda-\varepsilon} y d^{m-\varepsilon}\left(\mathfrak{A} y^{(m-\lambda+\varepsilon)}\right)$ ist. Die Summation nach $\lambda$ erstreckt sich auf alle Werthe von $\lambda$, welche gültige Glieder geben, eben so auch die Summation nach $\varepsilon$. Der Anblick der Formel für $\Omega_{\lambda}$ zeigt, dafs $\lambda$ die Werthe $0,1,2,3, \ldots$ bis $2 m, \varepsilon$ die Werthe $0,1,2, \ldots$ bis $m$ erhalten mufs; wenn jedoch $\lambda$ kleiner ist als $m$, so werden alle die Werthe von $\varepsilon$ ungultig, welche gröfser sind als $\lambda$. Wird noch für $\varepsilon, \lambda-m+\mu$ eingeführt, so folgt:

$$
\mathfrak{R}_{\lambda}=\sum_{\mu} m_{\varepsilon} \boldsymbol{m} \boldsymbol{n}_{\lambda-\varepsilon} y d^{m-\varepsilon}\left(\mathfrak{A} y^{(\mu)}\right),
$$

wo der Buchstabe $\varepsilon$ nur vorläufig zur Abkürzung für seinen Werth $\lambda-m+\mu$ beibehalten ist. Um nun den Factor $y$ unter das Differentialzeichen zu bringen, dient die bekannte Formel:

$$
y d^{n} v=\sum_{\nu}(-1)^{v} n_{\nu} d^{n-v}\left(y^{(v)} v\right)
$$

durch deren Anwendung $\mathfrak{R}_{2}$ folgende Gestalt erlangt:

$$
\mathfrak{R}_{\lambda}=\sum_{\mu} \sum_{\nu}(-1)^{\nu} \boldsymbol{m}_{\lambda-\ell} \boldsymbol{m} \boldsymbol{m}_{\varepsilon}(m-\varepsilon)_{\nu} d^{m-\varepsilon-\nu}\left(\mathfrak{A} y^{(\mu)} y^{(\nu)}\right) \text {. }
$$

Es ist aber immer $m_{\varepsilon}(m-\varepsilon)_{\nu}=m_{\nu}(m-\nu)_{\varepsilon}$; setzt man noch für $\varepsilon$ seinen Werth $\lambda-m+\mu$, so ist $m_{\lambda-\varepsilon}=m_{m-\mu}=m_{\mu}, m-\varepsilon-\nu=2 m-\lambda-\mu-\nu$; daher:

$$
\mathfrak{Q}_{\lambda}=\sum_{\mu} \sum_{\nu}(-1)^{\nu} m_{\mu} m_{\nu}(m-\nu)_{\lambda-m+\mu} d^{2 m-\lambda-\mu-\nu}\left(\mathfrak{A} y^{(\mu)} y^{(\nu)}\right) .
$$


Um diesen Werth auf das einfachste zu schreiben, setze ich noch $2 m-2 \lambda-\mu-\nu=q$ und bezeichne $\mathfrak{A} y^{(\mu)} y^{(\nu)}$ mit $\boldsymbol{Q}(\mu, \nu)$, so folgt:

$$
\mathfrak{\Omega}_{\lambda}=\sum_{\mu} \sum_{\nu}(-1)^{\nu} m_{\mu} m_{\nu}(m-\nu)_{\lambda-m+\mu} d^{q+\lambda} Q(\mu, \nu) .
$$

Dieser ohne alle Schwierigkeit sich darbietende Werth von $\mathbb{R}_{\lambda}$ gewinnt nun eine neue Gestalt, in welcher sich die $\mathfrak{B}$ leicht erkennen lassen, wenn man die Gleichung (A.) zur Verwandlung des Factors $(m-\nu)_{\lambda-m+\mu}$ verwendet. Selzt man nämlich in (A.) $m-\nu$ für $a, \lambda$ für $b, \lambda-m+\mu$ für $\nu$, so folgt:

daher:

$$
(\boldsymbol{m}-\nu)_{\lambda-m+\mu}=\sum_{j}(\lambda-j)_{j} f(\lambda-m+\nu-j, \lambda-m+\mu-j)
$$

$\mathfrak{\Omega}_{\lambda}=\sum_{j} \sum_{\mu} \sum_{\nu}(-1)^{\nu} m_{\mu} \boldsymbol{n}_{\nu}(\lambda-j)_{j} f(\lambda-m+\nu-j, \lambda-m+\mu-j) d^{q+\lambda} \boldsymbol{Q}(\mu, \nu)$,

wo die Summationen nach $j, \mu, \nu$ überhaupt alle gültigen Glieder umfassen. Aus diesem Ausdrucke nehme man das zu $j=0$ gehörige Glied heraus, nämlich $\sum_{\mu} \sum_{\nu}(-1)^{\nu} m_{\mu} m_{\nu} f(\lambda-m+\nu, \lambda-m+\mu) d^{q+\lambda} Q(\mu, \nu)$ und bezeichne es mit $d^{\lambda} \mathfrak{B}_{\lambda}$, indem man setzt:

$$
\mathfrak{B}_{\lambda}=\sum_{\mu} \sum_{\nu}(-1)^{\nu} m_{\mu} m_{\nu} f(\lambda-m+\nu, \lambda-m+\mu) d^{q} \boldsymbol{Q}(\mu, \nu),
$$

so wird, da durch Verwandlung von $\lambda$ in $\lambda-j, q=2 m-2 \lambda-\mu-\nu$ in $q+2 j$ übergeht:

$$
\mathfrak{B}_{\lambda-j}=\sum_{\mu} \sum_{\nu}(-1)^{\nu} m_{\mu} m_{\nu} f(\lambda-m+\nu-j, \lambda-m+\mu-j) d^{q+2 j} \boldsymbol{Q}(\mu, \nu),
$$

daher:

$$
d^{\lambda-2 j} \mathfrak{B}_{\lambda-j}=\sum_{\mu} \sum_{\nu}(-1)^{\nu} m_{\mu} m_{v} f(\lambda-m+\nu-j, \lambda-m+\mu-j) d^{q+\lambda} \boldsymbol{Q}(\mu, \nu) .
$$

Vergleicht man hiermit den vorstehenden Werth von $\mathfrak{l}_{\lambda}$, so folgt:

$$
\mathfrak{R}_{\lambda}=\sum_{j}(\lambda-j)_{j} d^{\lambda-2 j} \mathfrak{B}_{\lambda-j} \text {. }
$$

Oben war $\Delta+\mathfrak{\Omega}_{0} t=\sum_{2} \mathfrak{\Omega}_{\lambda} t^{(\lambda)}$; also ist:

$$
\Delta+\mathfrak{\Omega}_{0} t=\sum_{\lambda} \sum_{j}(\lambda-j)_{j} t^{(i)} d^{\lambda-2 j} \mathfrak{B}_{\lambda-j}
$$

oder wenn für $\lambda, l+j$ gesetzt wird:

$$
\Delta+\Omega_{0} t=\sum_{l} \sum_{j} l_{j} t^{(l+j)} d^{l-j} \mathfrak{B}_{l} .
$$

Es ist aber $\sum_{j} l_{j} t^{(l+j)} d^{l-j} \mathfrak{B}_{l}=d^{l}\left(\mathfrak{B}_{l} t^{(l)}\right)$; daher:

$$
\Delta+\mathfrak{\Omega}_{0} t=\sum_{l} d^{l}\left(\mathfrak{B}_{l} t^{(l)}\right) \text {. }
$$

Bemerkt man nun noch, dafs zufolge der obigen Werthe $\mathfrak{l}_{0}=\mathfrak{B}_{0}=y d^{m}\left(\mathfrak{A} y^{(m)}\right)$ 
ist, und zieht demgemäfs die Gleichung $\ell_{0} t=\mathfrak{B}_{0} t$ von der vorstehenden ab, so folgt:

$$
\Delta=\sum_{l} d^{l}\left(\mathfrak{B}_{l} t^{(l)}\right)
$$

wo die Summation sich auf alle gültigen Werthe von $l$ erstreckt, mit Ausnahme von $l=0$. Die Betrachtung des obigen Ausdrucks für $\mathfrak{B}_{\lambda}$ lehrt aber sofort, dafs darin $\mu$ und $\nu$ nur positive und nicht über $m$ hinausgehende Werthe erhalten dürfen, um gültige Glieder zu geben; da nun $f(a, i)=0$ ist, wenn $i$ negativ, so folgt, dafs $\mathfrak{B}_{\lambda}=0$ wird für ein negatives $\lambda$, da solches einen negativen Werth von $\lambda-m+\mu$ herbeifübren und mithin in $\mathfrak{B}_{\lambda}$ den Factor $f(\lambda-m+\nu, \lambda-m+\mu)=0$ machen würde.

Es läfst sich ferner leicht zeigen, dafs der obige Werth von $\mathfrak{B}_{\lambda}$ keine Glieder entbält, für welche $q$ negativ würde. Vertauscht man nämlich $\mu$ mit $\nu$, so bleibt $\boldsymbol{Q}(\mu, \nu)=\mathfrak{A} y^{(\mu)} y^{(\nu)}$ ungeändert, eben so auch $q=2 m-2 \lambda-\mu-\nu$; es gehört daher in $\mathfrak{B}_{\lambda}$ zu einerlei $\boldsymbol{Q}(\mu, \nu)$ und $\boldsymbol{q}$ der Factor:

$$
m_{\mu} m_{\nu}\left\{(-1)^{\nu} f(\lambda-m+\nu, \lambda-m+\mu)+(-1)^{\mu} f(\lambda-m+\mu, \lambda-m+v)\right\} \text {. }
$$

Dieser Factor ist aber nach einer früheren Bemerkung gleich Null, wenn $(\lambda-m+\mu)+(\lambda-m+\nu)=-q$ von Null verschieden und positiv ist; also kommt in der mit $\mathfrak{B}_{\lambda}$ bezeichneten Summe kein Glied mit negativem $q$ vor. Es bedarf kaum noch der Erwähnung, dafs derselbe Beweis auch gültig bleibt, wenn $\mu=\nu$ angenommen wird.

Es ist endlich noch zu zeigen, dafs der aufgestellte Werth von $\mathfrak{B}_{\lambda}$ auch der Bedingung entspricht, wonach für $\lambda>m, \mathfrak{B}_{\lambda}=0$ werden mufs. Dies ist aber augenscheinlich der Fall; denn wenn $\lambda>m$ ist, so wird $2 \lambda-2 m+\mu+\nu=-q$ positiv, weil $\mu$ und $\nu$ nur positive Werthe erhalten oder gleich Null sein können; da aber alle Glieder mit negativem $q$ hinwegfallen, wie so eben gezeigt worden, so folgt $\mathfrak{B}_{\lambda}=0$ für $\lambda>m$.

Demnach wird:

wo:

$$
\Delta=\sum_{l=1} d^{l}\left(\mathfrak{B}_{l} t^{(l)}\right)=d\left(\mathfrak{B}_{1} t^{\prime}\right)+d^{2}\left(\mathfrak{B}_{2} t^{\prime \prime}\right)+\cdots+d^{m}\left(\mathfrak{B}_{m} t^{(m)}\right)
$$

$\mathfrak{B}_{\lambda}=\sum_{\mu} \sum_{\nu}(-1)^{\nu} m_{\mu} m_{\nu}\left\{(m-\lambda-\nu)_{\lambda-m+\mu}+(m-\lambda-\nu-1)_{\lambda-m+\mu-1}\right\} d^{q}\left(\mathfrak{A} y^{(\mu)} y^{(\nu)}\right)$, und :

$$
q=2 m-2 \lambda-\mu-\nu>0 .
$$

Es sei $\lambda-m+\mu=k$, daher $m-\nu-\lambda=q+k$, so läfst der Werth von $\mathfrak{B}_{\lambda}$ sich auch also schreiben:

$\mathfrak{B}_{\lambda}=\sum_{q} \sum_{k}(-1)^{m-\lambda-q-k} m_{\lambda+q+k} m_{\lambda-k}\left\{(q+k)_{k}+(q+k-1)_{k-1}\right\} d^{q}\left(\mathfrak{A} y^{(m+k-\lambda)} y^{(m-\lambda-q-k)}\right)$. 
Die Summationen nach $q$ und $k$ erstrecken sich auf alle Werthe, welche gültige Glieder geben, also für $q$ von Null bis $m-\lambda$, für $k$ von Null bis zu der kleineren der beiden Zahlen $\lambda$ und $m-\lambda-q$.

Ich setze einige nach diesen Formeln berechnete Beispiele her. Es ist:

$$
\Delta=y d^{m}\left(\mathfrak{A}(y t)^{(m)}\right)-y t d^{m}\left(\mathfrak{A} y^{(m)}\right)=\sum_{\lambda=1}^{\lambda=m} d^{\lambda}\left(\mathfrak{B}_{\lambda} t^{(\lambda)}\right)
$$

für $m=1$ wird $\mathfrak{B}_{1}=\mathfrak{A} y y$.

$$
\begin{aligned}
& m=2 . \quad \mathfrak{B}_{1}=2 d . \mathfrak{A} y y^{\prime}+2 \mathfrak{A}\left(y y^{\prime \prime}-2 y^{\prime} y^{\prime}\right) . \quad \mathfrak{B}_{2}=\mathfrak{A} y y . \\
& \left.m=3 \text {. } \quad \mathfrak{B}_{1}=3 d^{2} \cdot \mathfrak{A} y y^{\prime \prime}-3 d \cdot \overline{\mathcal{A}\left(3 y^{\prime} y^{\prime \prime}-y y^{\prime \prime \prime}\right.}\right)+3 \mathfrak{A}\left(3 y^{\prime \prime} y^{\prime \prime}-2 y^{\prime} y^{\prime \prime \prime}\right) \text {. } \\
& \mathfrak{B}_{2}=3 d . \mathfrak{A} y y^{\prime}+3 \mathfrak{A}\left(2 y y^{\prime \prime}-3 y^{\prime} y^{\prime}\right) . \quad \mathfrak{B}_{3}=\mathfrak{A} y y \text {. } \\
& m=4 . \quad \mathfrak{B}_{1}=4 d^{3} \cdot \mathfrak{A} y y^{\prime \prime \prime}+4 d^{2} \cdot \overline{\mathfrak{A}\left(y y^{\mathrm{IV}}-4 y^{\prime} y^{\prime \prime \prime}\right)}+12 d \cdot \overline{\mathfrak{A}\left(2 y^{\prime \prime} y^{\prime \prime \prime}-y^{\prime} y^{\mathrm{IV}}\right)} \\
& \mathfrak{B}_{2}=6 d^{2} \cdot \mathfrak{A} y y^{\prime \prime}+12 d \cdot \frac{+\mathfrak{A}\left(y y^{\prime \prime \prime}-2 y^{\prime} y^{\prime \prime}\right)}{4}+4\left(3 y^{\prime \prime} y^{1 v}-4 y^{\prime \prime \prime} y^{\prime \prime \prime}\right) \text {. } \\
& +2 \mathscr{A}\left(y y^{\mathrm{Iv}}-16 y^{\prime} y^{\prime \prime \prime}+18 y^{\prime \prime} y^{\prime \prime}\right) \text {. } \\
& \mathfrak{B}_{3}=4 d . \mathfrak{A} y y^{\prime}+4 \mathfrak{A}\left(3 y y^{\prime \prime}-4 y^{\prime} y^{\prime}\right) . \quad \mathfrak{B}_{4}=\mathfrak{A} y y .
\end{aligned}
$$

Nach dem zweiten Satze Jacobis lăfst sich die Variation von :

$$
V=\frac{d F}{d y}-d \frac{d F}{d y^{\prime}}+d^{2} \frac{d F}{d y^{\prime \prime}}-\cdots+(-1)^{n} d^{n} \frac{d F}{d y^{(n)}}=V_{n},
$$

wo $\boldsymbol{F}=f\left(x, y, y^{\prime}, y^{\prime \prime}, \ldots y^{(n)}\right)$, auf folgende Gestalt bringen:

$$
\delta V_{n}=\mathfrak{A} \delta y+d\left(\mathfrak{A}_{1} \delta y^{\prime}\right)+d^{2}\left(\mathfrak{A}_{2} \delta y^{\prime \prime}\right)+\cdots+d^{n}\left(\mathfrak{A}_{n} \delta y^{(n)}\right) .
$$

Werden in der entwickelten Variation $\delta V_{n}$ sämmlliche Glieder, welche $\frac{d F}{d y^{(n)}}$ enthalten, von den übrigen abgesondert, so kann die Summe der letzteren passend durch $\left(\delta V_{n-1}\right)$ angedeutet werden, und setzt man noch zur Abkürzung $\frac{d F}{d y^{(n)}}=\varphi$, so ergiebt sich :

$$
\begin{gathered}
\delta \boldsymbol{V}_{n}=\left(\delta \boldsymbol{V}_{n-1}\right)+\frac{d \varphi}{d y} \delta y^{(n)}-d\left(\frac{d \varphi}{d y^{\prime}} \delta y^{(n)}\right) \\
+d^{2}\left(\frac{d \varphi}{d y^{\prime \prime}} \delta y^{(n)}\right)-\cdots+(-1)^{n-1} d^{n-1}\left(\frac{d \varphi}{d y^{(n-1)}} \delta y^{(n)}\right) \\
+(-1)^{n} d^{n}\left\{\frac{d \varphi}{d y} \delta y+\frac{d \varphi}{d y^{\prime}} \delta y^{\prime}+\cdots+\frac{d \varphi}{d y^{(n)}} \delta y^{(n)}\right\},
\end{gathered}
$$

oder :$$
d V_{n}-\left(\delta V_{n-1}\right)=(-1)^{n} \sum_{\mu=0}^{\mu=n-1} d^{\mu} U_{n-\mu}+(-1)^{n} d^{n}\left(\frac{d \varphi}{d y^{(n)}} \delta y^{(n)}\right),
$$ 
wo gesetzt ist:

$$
\boldsymbol{U}_{n-\mu}=d^{n-\mu}\left(\frac{d \varphi}{d y^{(\mu)}} \delta y^{(\mu)}\right)+(-1)^{n-\mu} \frac{d \varphi}{d y^{(\mu)}} \delta y^{(n)}
$$

Wird hier $\frac{d \varphi}{d y^{(\mu)}}=\frac{d^{2} F}{d y^{(\mu)} d y^{(n)}}$ mit $v$ und $\delta y^{(\mu)}$ mit $w$ bezeichnet, so erhält man:

$$
\boldsymbol{U}_{n-\mu}=\boldsymbol{d}^{n-\mu}(\boldsymbol{v} \boldsymbol{w})+(-\mathbf{1})^{n-\mu} \boldsymbol{v} \boldsymbol{w}^{(n-\mu)},
$$

also nach Formel (B.), wenn durch $(n-\mu)^{\prime}$ die grölste in $\frac{n-\mu}{2}$ enthaltene ganze Zahl angedeutet wird:

$$
U_{n-\mu}=\sum_{i=0}^{i=(n-\mu)^{\prime}} f(i+\mu-n, i) d^{i}\left(v^{(n-\mu-2 i)} w^{(i)}\right)
$$

Der Werth von $f(i+\mu-n, i)$ ist:

$$
\frac{(n-\mu)(n-\mu-i-1)(n-\mu-i-2) \ldots(n-\mu-2 i+1)}{1.2 .3 \ldots i} \text {. }
$$

Stellt man endlich für $v$ und $w$ ihre so eben gesetzten Werthe wieder her, so folgt:

$$
d^{\mu} U_{n-\mu}=\sum_{i=0}^{i=(n-\mu)^{r}} f(i+\mu-n, i) d^{\mu+i}\left(d^{n-\mu-2 i}\left(\frac{d^{2} F}{d y^{(\mu)} d y^{(n)}}\right) \delta y^{(\mu+i)}\right),
$$

wodurch $\delta \boldsymbol{V}_{n}-\left(\delta \boldsymbol{V}_{n-1}\right)$ und damit $\delta \boldsymbol{V}_{n}$ selbst auf die verlangte Gestalt gebracht ist.

Dorpat, im December 1857. 